\title{
No velocity-kicks are required to explain large-distance offsets of Ca-rich supernovae and short-GRBs
}

\author{
Hagai B. Perets ${ }^{\circledR 1 \star}$ and Paz Beniamini ${ }^{\circledR 2,3}$ \\ ${ }^{1}$ Physics Department, Technion - Israel Institute of Technology, Haifa 3200003, Israel \\ ${ }^{2}$ Theoretical Astrophysics, Walter Burke Institute for Theoretical Physics, Mail Code 350-17, Caltech, Pasadena, CA 91125, USA \\ ${ }^{3}$ Astrophysics Research Center of the Open University (ARCO), The Open University of Israel, P.O Box 808, Ra'anana 43537, Israel
}

Accepted 2021 March 15. Received 2021 March 15; in original form 2021 January 26

\begin{abstract}
Environments of supernovae ( $\mathrm{SNe}$ ) and gamma-ray bursts (GRBs) link their progenitors to the underlying stellar population, providing critical clues for their origins. However, various transients including Ca-rich SNe and short-GRBs, appear to be located at remote locations, far from the stellar population of their host galaxy, challenging our understanding of their origin and/or physical evolution. These findings instigated models suggesting that either large velocity-kicks were imparted to the transient progenitors, allowing them to propagate to large distances and attain their remote locations; or that they formed in dense globular-clusters residing in the haloes. Here we show that instead, large spatial-offsets of such transients are naturally explained by observations of highly extended stellar populations in (mostly early-type) galaxy haloes, typically missed since they can only be identified through ultra-deep/stacked images. Consequently, no large velocity kicks, nor halo globular-cluster environments are required in order to explain the origin of these transients. These findings support thermonuclear explosions on white-dwarfs, for the origins of Ca-rich SNe progenitors, and the existence of small (or zero) kick-velocities given to short-GRB progenitors. Furthermore, since stacked/ultra-deep imaging show that early-type galaxies are more extended than late-type galaxies, studies of transients' offset-distribution (e.g. type Ia SNe or FRBs) should account for host galaxy-type. Since early-type galaxies contain older stellar populations, transient arising from older stellar populations would have larger fractions of early-type hosts, and consequently larger fractions of large-offset transients. In agreement with our results for short-GRBs and Ca-rich SNe showing different offset distributions in early versus late-type galaxies.
\end{abstract}

Key words: gamma-ray burst: general - stars: neutron - fast radio bursts - gamma-ray bursts - neutron star mergers - transients: supernovae.

\section{INTRODUCTION}

The host galaxies and the positions of explosive transients inside their hosts hold critical information about their progenitors. The progenitors of supernovae ( $\mathrm{SNe}$ ) and gamma-ray bursts (GRBs) can be potentially linked to the underlying stellar populations in their close environments.

For example, the star formation history of the host galaxy of a given transient provides a statistical inference of the age of the progenitor of the transient. Given a statistical sample of transients of a specific type, one can even infer the delay time distribution for that type of explosive events (e.g. Maoz, Mannucci \& Nelemans 2014). Such inference has a long history, with Baade noting already early-on that type II SNe explode in early-type (spiral) galaxies, while type I SNe explode both in early- and late-type galaxies, providing the first clue of our current understanding of type II SNe arising from corecollapse $\mathrm{SNe}$, while type I SNe arising from both core-collapse (most $\mathrm{Ib}, \mathrm{Ic} \mathrm{SNe}$ ) and thermonuclear $\mathrm{SNe}$ (Ia SNe). The local environments of transients inside their host galaxy provide further information on

^E-mail: hperets@physics.technion.ac.il the stellar populations of their progenitors, their metallicities and typical lifetimes.

In some cases, transients were found to be located in a different environment than expected from their theorized progenitors. Those cases assisted in identifying and eventually classifying such objects as part of a new class of objects arising from different type of progenitors. Two examples, which are also the focus of this study are short-GRBs (sGRBs) and Ca-rich SNe. For instance, long GRBs are typically observed in young environments in late-type type starforming galaxies and close to star-forming regions in these galaxies, pointing to their likely origin from massive young stars (Bloom, Kulkarni \& Djorgovski 2002). In contrast, sGRBs were found both in late and in early-type galaxies, and in many of which far from any star-forming region (Fong \& Berger 2013; Fong et al. 2017). This was found to be consistent with the idea of sGRBs arising from the mergers of neutron stars (NSs; and confirmed through the recent multi-messenger gravitational-wave and electromagnetic counterpart GRB 170817A (Abbott et al. 2017)). Similarly, some faint type Ib $\mathrm{SNe}$ were found in late-type galaxies, and far from star-forming regions, in contrast with the expected massive progenitors for corecollapse SNe typically thought to produce type Ib SNe (Perets et al. 2010). This gave rise to the identification of a new class of Ca-rich, 
faint type $\mathrm{Ib} \mathrm{SNe}$, suggested to arise from thermonuclear He-rich explosions of white-dwarfs (Perets et al. 2010), as we further discuss below.

The early-type host galaxies and the sometimes large distances from any star-forming regions of both sGRBs and Ca-rich SNe point to old progenitors for a significant fraction of these transients. This is consistent with the suggested NS mergers and thermonuclear WD explosions which can indeed have very long delay-time since the formation of their progenitor stars. However, in many cases, not only were these transients far from any star-forming regions, but they also appeared to be located very far from their host galaxies (Perets et al. 2010; Kasliwal et al. 2012; Fong \& Berger 2013; Fong et al. 2017; Lunnan et al. 2017; De et al. 2020). It was therefore suggested by several studies that the remote locations of such transients could be explained either by large velocity kicks given to their progenitors ((Lyman et al. 2014; Foley 2015; Lyman et al. 2016) for Ca-rich $\mathrm{SNe}$;(Fong \& Berger 2013) for sGRBs), or that the progenitors form in dense globular clusters, since many of those reside in the haloes (Yuan et al. 2013; Sell et al. 2015; Shen, Quataert \& Pakmor 2019). In the former scenario, the post-kick progenitors could then propagate through their host galaxies over hundreds of Myrs attaining remote positions at the time of their explosions. In particular, the observed high velocities of pulsars point to natal kicks given to NSs upon their formation. Since sGRBs are produced through the merger of NSs, it was thought plausible that sGRBs could be found at large offsets from their host galaxies. However, as we discuss below, it is debated whether indeed sGRB progenitors receive such large kicks.

Following a similar reasoning it was suggested that many of the progenitors of $\mathrm{Ca}$-rich $\mathrm{SNe}$ receive some large velocity kicks, either due to interactions of their progenitors with massive black holes in galaxy nuclei leading to their ejection as hypervelocity stars, or possibly due to their progenitors containing a NS which received a large natal kick.

Already a few years ago, we pointed out that the recent findings that the stellar haloes of early-type galaxies are far larger than previously thought, would give rise to transients from old stellar population at these haloes (Perets 2014). Since the underlying stellar populations can only be seen through deep imaging and/or stacking, transients arising from the halo stellar population in such galaxies would appear to be located far from the stellar populations of their host galaxies, while, in fact, they formed in-situ from stars in the difficult-to-observe underlying population.

Here we follow this suggestion and study the detailed distributions of Ca-rich SNe and SGRBs, while dividing them between early- and late-type galaxies, which posses very different underlying stellar haloes. As we discuss in the following, we find that the large offsets observed for both sGRBs and Ca-rich SNe can indeed be naturally explained without invoking any (or large) natal kicks, and their progenitors could have been formed in-situ.

The paper is structured as follows. We first discuss the distribution of the stellar masses in early- versus late-type galaxies (Section 2), we then analyse the offsets distributions of short Ca-rich $\mathrm{SNe}$ and sGRBs (Section 3). We discuss our results and their implications for Ca-rich SNe progenitors and the natal kicks given to SGRB progenitors in Section 4, and summarize (Section 5).

\section{THE DISTRIBUTION OF STELLAR MASS IN GALAXY HALOES}

Traditionally, only small fractions of the stellar mass in galaxies were directly observed at large offsets (10-100 kpcs; a region which we term here the galaxy halo) from their centres. The halo of the Milky- way galaxy, for example, contains only 1-2 percent of the stellar mass in the Galaxy. For this reason, identification of transients at large offsets from the centres of their host galaxies was typically suggested to originate from some type of velocity kicks given to their progenitors, as such stellar progenitors were thought unlikely to exist at such large offsets (especially when such large offsets are frequent for some specific type of transients). In particular, large kick velocities could allow them to migrate to large distances from their original birth place in the inner parts of the galaxy and/or their disc components, where most of the stars were expected to exist.

However, the distribution of stellar mass in galaxy haloes, far from the nucleus is difficult to measure given the low surface brightness in these regions. The direct measurement of the stellar mass in galaxy haloes for any single galaxy is notoriously difficult and could significantly underestimate the stellar mass in the halo. In fact, galaxy formation simulations have suggested that many galaxy haloes should in fact contain significant fractions of the stellar mass (e.g. Sanderson et al. 2018, and references therein).

Only in the last few years did the stellar mass in the haloes of galaxies were measured for different types and different masses of galaxies, over large statistical samples, allowing to infer the haloes stellar masses. Such measurements were done through the use of stacking of observations of many galaxies belonging to the same type and mass range (D'Souza et al. 2014), or through deep imaging of single galaxies using the Hyper Suprime-Cam (Huang et al. 2018). These studies have shown that early-type galaxies (ellipticals and S0 galaxies; more centrally concentrated galaxies) have 30-70 per cent of their stellar mass in the halo (with a monotonically rising trend with galaxy mass), while late type, spiral galaxies have only 0 25 per cent of their stellar mass in the halo (consistent with the case of the Milky-Way).

The stellar population in early-type galaxies is old, typically at least 9-10 Gyr old (e.g. Gallazzi et al. 2005), and no star formation is typically observed in galaxy haloes. Taken together, one would expect transients arising from young stellar populations to be observed in the discs of spiral galaxies, and not in galaxy haloes, and thereby generally have small $(<10 \mathrm{kpc})$ offsets. In contrast, transients arising from old stellar progenitors should be observed with comparable numbers in the halo and the central parts of earlytype galaxies, and mostly in the discs and central parts of late-type galaxies (since only a small fraction of the old stellar population resides in the halo).

As we discuss in what follows, these recent developments in understanding the distribution of the stellar mass in galaxies have far reaching implications for the interpretations of the offsets' distribution of transients, and its ramifications regarding the progenitors of such transients, and the physical processes involved in their evolution. In particular, observations of large offsets for some types of transients such as sGRBs and Ca-rich faint supernovae should not imply the need for some natal velocity kicks for their progenitors, but rather that such large-offset transients arise from old stellar populations, and were not likely to receive large natal kick and then migrate to their observed position. As we show below, the correlation between the type of host galaxies and the offset distribution of the transients corroborates this.

\section{CA-RICH SUPERNOVAE AND SGRB OFFSETS' DISTRIBUTIONS}

In the following, we study the offset distribution of the two types of transients, Ca-rich SNe and sGRBs. The samples are summarized in Tables 1 and 2. Their cumulative distributions, divided between 
Table 1. Sample of Ca-rich SNe used in this work.

\begin{tabular}{lcc}
\hline Supernova & Offset $(\mathrm{kpc})$ & Galaxy type \\
\hline $2000 \mathrm{ds}$ & 3.77 & Early \\
$2001 \mathrm{co}$ & 7.07 & Late \\
$2003 \mathrm{H}$ & 8.73 & Late \\
$2003 \mathrm{dg}$ & 1.66 & Late \\
$2003 \mathrm{dr}$ & 2.65 & Late \\
$2005 \mathrm{E}$ & 24.27 & Early \\
$2005 \mathrm{cz}$ & 2.12 & Early \\
$2007 \mathrm{ke}$ & 16.71 & Early \\
$2010 \mathrm{et}$ & 37.64 & Early \\
$2012 \mathrm{hn}$ & 6.73 & Early \\
PTF11bij & 34.42 & Early \\
PTF11kmb & 150.05 & Early \\
$2016 \mathrm{hgs}$ & 5.9 & Late \\
$2018 \mathrm{ckd}$ & 19.08 & Early \\
$20181 \mathrm{qo}$ & 15.46 & Early \\
$20181 \mathrm{qu}$ & 26.70 & Early \\
$2018 \mathrm{gwo}$ & 8.56 & Early \\
$2018 \mathrm{kjy}$ & 6.35 & Early \\
$2019 \mathrm{ehk}$ & 1.8 & Late \\
$2019 \mathrm{hty}$ & 8.73 & Early \\
$2019 \mathrm{pxu}$ & 17.56 & Late \\
\hline
\end{tabular}

Table 2. Sample of sGRBs used in this work.

\begin{tabular}{lccc}
\hline GRB & Type & $\log _{10}\left(M_{*} / M_{\odot}\right)$ & Offset $(\mathrm{kpc})$ \\
\hline $050509 \mathrm{~B}$ & Early & $11.08 \pm 0.03$ & $63.7 \pm 12.2$ \\
050709 & Late & $8.66 \pm 0.07$ & $3.64 \pm 0.027$ \\
050724 & Early & $10.64 \pm 0.05$ & $2.63 \pm 0.079$ \\
$051221 \mathrm{~A}$ & Late & $8.61 \pm 0.64$ & $2.18 \pm 0.19$ \\
$060502 \mathrm{~B}$ & Early & $\sim 11.8$ & $73 \pm 19$ \\
061006 & Late & $10.43 \pm 0.23$ & $1.3 \pm 0.24$ \\
$070429 \mathrm{~B}$ & Late & $\sim 10.4$ & 4.7 \\
$070714 \mathrm{~B}$ & Late & $\sim 9.4$ & $12.21 \pm 0.87$ \\
$070724 \mathrm{~A}$ & Late & $\sim 10.1$ & $5.46 \pm 0.14$ \\
070809 & Early & $\sim 11.4$ & $33.22 \pm 2.71$ \\
071227 & Late & $\sim 10.4$ & $15.5 \pm 0.24$ \\
$080905 \mathrm{~A}$ & Late & $10.3 \pm 0.3$ & $17.96 \pm 0.19$ \\
090426 & Late & - & $0.45 \pm 0.25$ \\
090510 & Late & $\sim 9.7$ & $10.37 \pm 2.89$ \\
090515 & Early & $\sim 11.2$ & $75.03 \pm 0.15$ \\
$100117 \mathrm{~A}$ & Early & $\sim 10.3$ & $1.32 \pm 0.33$ \\
$111117 \mathrm{~A}$ & Late & $9.9 \pm 0.2$ & $8.5 \pm 1.7$ \\
$120804 \mathrm{~A}$ & Late & $\sim 10.8$ & $2.2 \pm 1.2$ \\
$130603 \mathrm{~B}$ & Late & $\sim 9.7$ & $5.21 \pm 0.17$ \\
$150101 \mathrm{~B}$ & Early & $10.85 \pm 0.12$ & $7.35 \pm 0.07$ \\
$170817 \mathrm{~A}$ & Early & $10.65 \pm 0.03$ & $2.12 \pm 0.001$ \\
$181123 \mathrm{~B}$ & Late & $10.24 \pm 0.16$ & $5.08 \pm 1.38$ \\
200522A & Late & $9.44 \pm 0.02$ & $1.07 \pm 0.27$ \\
\hline
\end{tabular}

early- and late-type galaxies are shown in Fig. 1. Descriptions of the two samples are summarized below.

\subsection{Ca-rich supernovae sample}

Our current sample of Ca-rich $\mathrm{SNe}$ offsets is based on the sample from our original characterization study (Perets et al. 2010) based on $\mathrm{SNe}$ from the LOSS and CCCP surveys, and the additional Ca-rich SNe discovered by the PTF/iPTF (Kasliwal et al. 2012; Lunnan et al. 2017; De et al. 2018), PESSTO (Valenti et al. 2014) and ZTF (De et al. 2020; Jacobson-Galán et al. 2020, 2021) surveys. The SNe offsets are taken from these studies, and summarized in Table 1; the cumulative

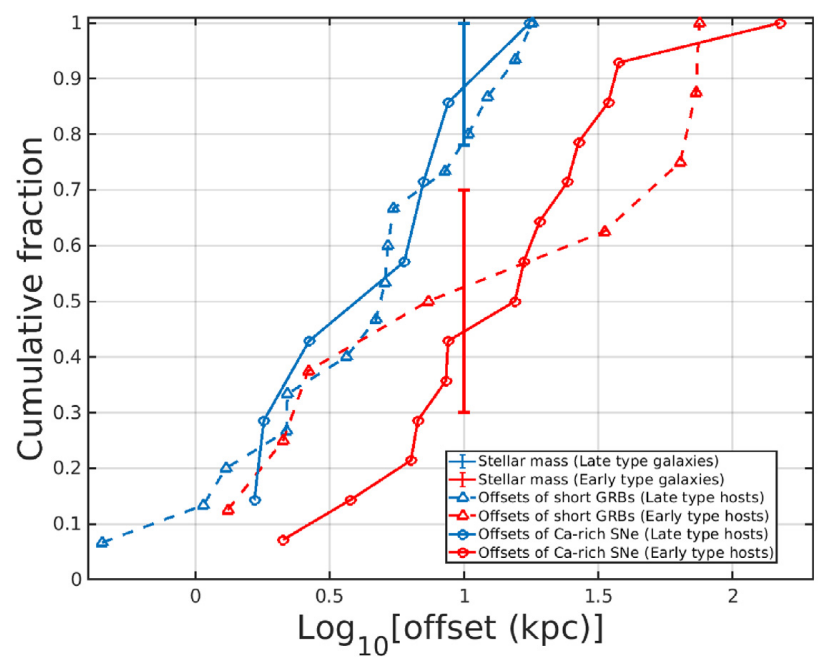

Figure 1. The cumulative offset distributions of sGRBs and Ca-rich supernovae in early- and late-type galaxies. Note that these host galaxies range in mass and size. The offset distributions of both SGRBs and Ca-rich SNe differ between the early-type host galaxies and the late-type host galaxies. The offset distribution is far more extended in the former, compared with latter, consistent with the far more extended distribution of the halo stellar mass in early-type galaxies, compared with late-type galaxies, and consistent with the observed stellar masses in these types of galaxies (vertical lines, from D'Souza et al. 2014; showing a range, corresponding to the range of galaxy masses, with more massive galaxies having higher halo stellar mass fractions). These results suggest an in-situ formation for the progenitors of these transients, without the need of any natal velocity kicks, nor the existence of globular clusters.

offset distributions divided between early- and late-type galaxies can be found in Fig. 1. The error bars on the offsets of these SNe are typically not given but are comparable to the least significant digit. We do not consider one Ca-rich SN, PTF12bho suggested to explode in the intracluster medium (since a large fraction of the stellar mass in galaxy clusters reside in the intracluster medium (e.g. Gonzalez, Zaritsky \& Zabludoff 2007; Da Rocha, Ziegler \& Mendes de Oliveira 2008, and references therein); the existence of one such SN in our sample could be expected). For the majority of the $\mathrm{SNe}$, the host galaxy is the closest galaxy and can be well identified. In two cases (PTF11 kmb and PTF11bij) we followed the suggested host and offset identification in the discovery papers, although several galaxies were found nearby, and the exact identification could be debated. These might even relate to the intracluster regions where they were found. Indeed, $\sim 15-20$ per cent of type Ia SNe in clusters are found in the intracluster medium far from any host galaxy (Gal-Yam et al. 2003; Sand et al. 2011).

We note that various $\mathrm{SNe}$ with only partial similarities to faint, type Ib, Ca-rich SNe characteristics were discussed in the literature (see e.g. De et al. 2020; Jacobson-Galán et al. 2020 for recent overviews, and references therein); these are not considered in our sample of bona-fide Ca-rich SNe). In some cases SNe we identified at late times post-peak (e.g. 2000ds, 2001co, 2003H, 2003dg, 2003dr) and therefore could, in principle, be related to some peculiar corecollapse type IIb SNe (e.g. see discussion of a possible core-collapse origin of iPTF 15eqv and SN 2019ehk, by Milisavljevic et al. (2017), De et al. (2021)). However, SN 2000ds host is an earlytype galaxy, and no clear star-forming regions were found close to 2000ds (Maund \& Smartt 2005), nor did the environment of SNe 2003H, 2001co, 2003dg or 2003dr (Lyman et al. 2014, 2016) show 
clear evidence for star formation. Therefore, current observations do not provide support for such an interpretation and we therefore assume that all of these are bona-fide Ca-rich $\mathrm{SNe}$.

\subsection{Short gamma-ray bursts sample}

The offsets of sGRBs have been extensively studied by various authors. We have compiled an up-to-date list of sGRBs with published offsets and galaxy types from (Fong et al. 2017; Gompertz, Levan \& Tanvir 2020; Paterson et al. 2020; O'Connor et al. 2021; Nugent et al. 2020; Fong et al. 2021). We limit the sample to those bursts with well determined offsets (i.e. error in offset determination of $\lesssim 50$ per cent and excluding bursts with ambiguity in the host galaxy determination). Our sample consists of 8 sGRBs in earlytype galaxies and 15 sGRBs in late-type galaxies and is listed in table 2.

In Fig. 1, we plot the cumulative offset distribution of sGRBs in early- and late-type galaxies. Large offsets occur predominantly in sGRBs associated with early-type galaxies. Indeed, already with the limited sample size available at the present time, the offset distributions in early- and late-type galaxies can be ruled out as being drawn from the same population at a level of 90 per cent confidence. This is inconsistent with the hypothesis that the offsets are dominated by strong kicks, in which case, the observed offsets (and especially for the large offset systems) will become independent of the galaxy type.

In principle, if we had deep imaging for each of the short GRBs or Ca-rich $\mathrm{SNe}$ ) hosts, we would have been able to normalize the offsets of the transients with the light and mass distribution. However, as we emphasized above, shallow observations of for example the halflight radii of galaxies do not correctly capture their underlying stellar mass distribution, and furthermore, the half-light radii are biased by the measured flux and thereby biased by the exposure time and the galaxy distance. We find that using such normalization (not shown) does indeed decrease the differences between the offset distributions of early- and late-type galaxies for the short GRB sample and even make them consistent with each other (there are not sufficient late-type galaxy hosts with measured half-light radii in SDSS for the Ca-rich sample as to make similar comparison). However, we again emphasize that using such approach is potentially problematic without deep imaging, and we therefore warrant against the simple use of such approach without careful accounting for biases, which in most cases is not possible with existing observations.

\section{DISCUSSION}

\subsection{The progenitors of Ca-rich supernovae}

Ca-rich SNe are faint (> - 16(-16.5) B(R) Mag) type Ib SNe, with strong $\mathrm{Ca}$ to $\mathrm{O}$ line ratio typically found in old stellar environments (Perets et al. 2010). A ratio of $[\mathrm{Ca} \mathrm{II]/[O} \mathrm{I]}>2$ was suggested as plausible delimiter (e.g. Milisavljevic et al. 2017). The prototype SN $2005 \mathrm{E}$ was found in the halo of an early type, S0 galaxy at a large offset ( $\sim 23 \mathrm{kpc})$ from its host galaxy center. At the time, all type Ib $\mathrm{SNe}$ were thought to arise from the core collapse of a massive star. We therefore initially thought that SN 2005E progenitor was a massive star that formed in the galaxy nucleus (where gas and star formation might exist even in an early-type galaxy), and was later ejected as a hypervelocity star following an interaction with the central massive black hole. It then propagated in the galaxy and exploded in its observed remote location in the galaxy halo. Although potentially possible, our analysis showed that the likelihood of observing an event from such a scenario was highly unlikely (Perets et al. 2010).

After collecting and characterizing a sample of such $\mathrm{SNe}$, we found many of them to explode in old stellar environments, and some at large offsets similar to SN 2005E. Together with our finding of inferred low ejecta-mass, low energy, low velocities and low ${ }^{56} \mathrm{Ni}$ mass, as well as potentially large abundances of intermediate elements (and the lack of any star-forming regions close-by) we concluded that Ca-rich SNe likely constitute a novel type of SN explosion arising from a thermonuclear He-rich explosion on a relatively old white dwarf (Perets et al. 2010). We therefore suggested that this scenario well explains the properties of these SNe, and the existence of such type Ib SNe in old stellar environments such as early-type galaxies and galaxy haloes (Perets et al. 2010; Perets et al. 2011; Waldman et al. 2011; Kasliwal et al. 2012; Lyman et al. 2013; Perets 2014).

The majority of Ca-rich SNe were found in old stellar populations in early-type galaxies (e.g. Perets et al. 2010; De et al. 2020) it was therefore expected that the locations and offsets of such $\mathrm{SNe}$ in their host galaxies would follow the old stellar populations in these galaxies. However, many of the SNe both in our original (Perets et al. 2010) sample and additional Ca-rich SNe identified later on (Kasliwal et al. 2012; De et al. 2020) were found at large offsets from their host galaxies (see Table 1 and Fig. 1), where very little, if any, stellar population was thought to exist.

As we first briefly suggested in Perets (2014) and now explore in detail in this study, the large offsets are in fact, a natural outcome from the existence of extended stellar haloes of (mostly) early-type galaxies (D'Souza et al. 2014; Huang et al. 2018), as discussed in Section 2.

As can be seen in Fig. 1, The offsets distribution in early-type and late galaxies show a different behaviour, with most of the SNe in late galaxies residing in the central $(<10 \mathrm{kpc})$ parts, while most of the $\mathrm{SNe}$ in early-type galaxies residing in the halo $(>10 \mathrm{kpc})$, nicely consistent with the stellar mass fractions for late- and early-type galaxies, respectively, as found in observations (D'Souza et al. 2014; Huang et al. 2018). In fact, in retrospect, the large offset distribution of these $\mathrm{SNe}$, and its difference in early- versus late-type galaxies (first shown by us here in Fig. 1) anticipated these findings.

The perplexing findings of large offsets instigated several studies suggesting that the remote locations of such transients could be explained either by large velocity kicks given to their progenitors either because their progenitors were NSs receiving a natal-kick at birth.

Yuan et al. (2013) and later Sell et al. (2015) and Shen et al. (2019) suggested that the extended distribution of the locations of Ca-rich is consistent with the distribution of globular clusters distribution. However, photometric searches for globular clusters close to the positions of known Ca-rich transients have generally been unsuccessful (Lyman et al. 2014). Furthermore, the relatively high inferred rate of Ca-rich SNe (5-15 per cent of the Ia SNe rate; Perets et al. 2010; De et al. 2020) could be difficult to explain with a globular cluster origin, given the low mass fraction in globular clusters.

Others suggested a large velocity kick is imparted to the progenitors of Ca-rich SNe (Lyman et al. 2014; Foley 2015; Lyman et al. 2016). Such scenarios are difficult to reconcile with the suggested WD merger/He-accretion origins of Ca-rich SNe (Perets et al. 2010; Shen et al. 2010; Waldman et al. 2011; Dessart \& Hillier 2015; Perets et al. 2019). They are also inconsistent with the expected rates and delay time distribution NS-WD mergers origins (Toonen et al. 2018; Zenati, Perets \& Toonen 2019), suggested as progenitors for Ca-rich 
SNe (Metzger 2012), and with the ejection rates of hypervlocity stars by massive black holes (Perets et al. 2010). Furthermore, as we showed here, the offsets in early-type galaxies appear to extend farther than in late-type galaxies, raising an extra challenge to a kick origin which relies on the micro-physics of the progenitor rather than on the galaxy macro-physics.

The results shown here therefore reconcile the difficulties and inconsistencies inferred form the large sample of Ca-rich $\mathrm{SNe}$ with large offsets. We find the large apparent offsets of Ca-rich $\mathrm{SNe}$ from the underlying stellar distribution is just an artefact of the low-surface stellar brightness in galaxy haloes, which is difficult to resolve with regular imaging, and requires the use of stacked or deep imaging. We conclude that $\mathrm{Ca}$-rich $\mathrm{SNe}$ mostly arise from an old stellar population, explaining the large fraction of early-type galaxies hosts. The high frequency of early-type hosts and the large extended stellar haloes of such galaxies explain the large offset distribution among Ca-rich $\mathrm{SNe}$.

We note that in early-type galaxies the fraction of Ca-rich $\mathrm{SNe}$ in the central 2-3 kpc appears to be lower than that of sGRBs in such galaxies. This might only reflect the low-statistics, but it is important to note that one should expect lower detection rates in the denser inner regions of galaxies, especially for very subluminous SNe such as Ca-rich SNe, as already suggested by us (Perets 2014), i.e. we expect significant Shaw's selection bias (Shaw 1979) for such SNe.

Finally, early-type galaxies have higher metallicities than late-type galaxies (e.g. Li et al. 2018). We therefore raise the possibility that Ca-rich SNe progenitors originate from high metallicity environments, rather than low-metallicities as suggested by us and others in the past (Yuan et al. 2013; Perets 2014; Shen et al. 2019). However, it is difficult, at this point, to disentangle the metallicities aspect of finding Ca-rich $\mathrm{SNe}$ in mostly early-type galaxies from the long delay times for Ca-rich $\mathrm{SNe}$ which bias towards such galaxies. Detailed study of the host metallicities for Ca-rich $\mathrm{SNe}$ has yet to be done, and could potentially resolve this issue.

\subsection{The kick velocity of sGRB progenitors}

The binary neutron star (BNS) merger origin for sGRBs has been suggested decades ago (Goodman 1986; Eichler et al. 1989). It received a conclusive proof with the detection of GRB 170817, a GRB accompanying a GW trigger from a BNS merger (Abbott et al. 2017). Since BNSs receive kicks when the second star in the binary collapses to a neutron star, they could potentially travel significantly before merging and producing an sGRB. An estimation of these kicks, merger delay times and offset distances can be obtained from the Galactic population of BNSs.

Shortly after the discovery of the double pulsar J0737-3039 (Burgay et al. 2003), the orbital parameters of this system led Piran \& Shaviv (2004), Piran \& Shaviv (2005) to suggest that the younger pulsar in the system, pulsar B, must have formed with very small mass ejection and with virtually no kick velocity. The system had a very small eccentricity and it was located in the Galactic plane. The small eccentricity suggested a small mass ejection, unless a strongly fine-tuned kick was given to the system. However, the latter would have led to a large CM velocity and the location of the system in the Galactic plane indicated that at least the vertical (out of the plane) component of the velocity of the system was low. The very small proper motion predicted by Piran \& Shaviv (2004), Piran \& Shaviv (2005) was confirmed within a year by pulsar timing observations (Kramer et al. 2006). Dall'Osso, Piran \& Shaviv (2014) refined the original estimates, using the observed proper motion and other parameters of the system. They confirmed the earlier expectations

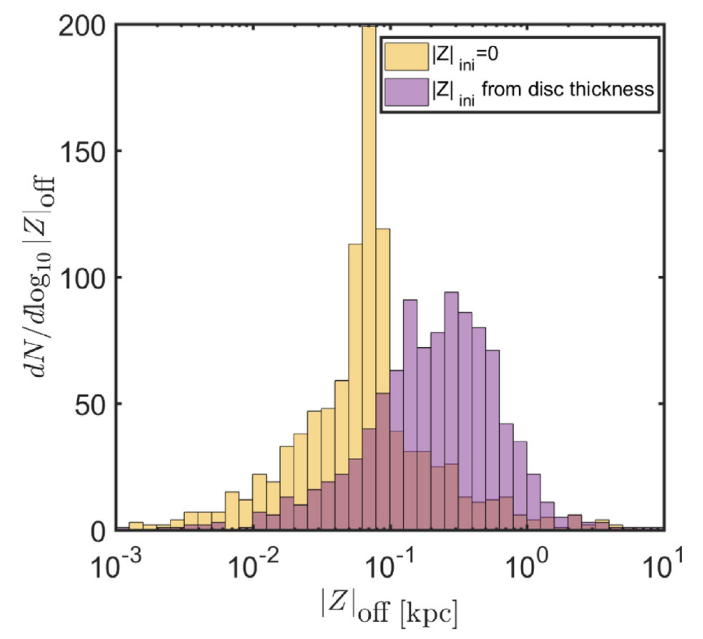

Figure 2. Results of a Monte Carlo simulation calculating the vertical offsets, $|z|_{\text {off }}$, of BNS systems from the Galactic plane at the time of merger (taking into account constraints on kicks and time to merger, consistent with constraints from the Galactic population, see Section A for details). We show results with the initial vertical offsets, $|z|_{\text {ini }}$, either in the Galactic plane (yellow) or following the disc thickness (purple). The results demonstrate that the offsets induced by kicks alone are typically $\lesssim 0.1 \mathrm{kpc}$, and suggest that the vertical offset distribution at merger is dominated by the initial vertical spread.

and have shown that in this system, the older pulsar (A) also most likely formed in this way.

Later on, Beniamini \& Piran (2016) have shown that out of 10 BNS systems observed in the Galaxy that do not reside in globular clusters, between six and seven must have been formed by collapses involving the release of a small amount of mass $\Delta M \lesssim 0.5 M_{\odot}$ and imparting only a very weak kick $v_{k} \lesssim 30 \mathrm{~km} / \mathrm{s}$, corresponding to changes in the center of mass velocity of the BNS of $\Delta v_{\mathrm{cm}} \lesssim 10 \mathrm{kms}^{-1}$. The other BNS systems require more typical core-collapse $\mathrm{SNe}$ with an ejected mass of one to a few solar masses and kicks of a few hundred km/sec. Since the publication of Beniamini \& Piran (2016), four new BNS systems have been reported: J1913+1102, J17571854, J1411-2551, J1946 + 2052 (Lazarus et al. 2016; Martinez et al. 2017; Cameron et al. 2018; Stovall et al. 2018). Three out of these four have small eccentricities $e=0.06,0.09,0.17$, suggesting a weak collapse origin and only one has $e=0.6$. This ratio is in a nice agreement with the earlier expectations and suggests that the result is not dominated by a statistical fluctuation. Furthermore, these conclusions were independently reproduced by Tauris et al. (2017). Overall, approximately $2 / 3$ of BNS systems observed so far require weak collapses with small kicks to account for their observed orbits.

With the small center of mass kicks mentioned above, BNS systems cannot easily break away from their host galaxy's potential and travel to large offsets. An illustrative example is given by the Milky Way. Assuming BNS systems are born close to the Galactic disc, and applying the small kicks mentioned above, will induce vertical oscillations above the Galactic plane, with a maximal length scale of $z_{\text {typ }} \sim \Delta v_{\mathrm{cm}} P_{z} / 2 \pi \sim 0.08 \mathrm{kpc}$, where $P_{z} \sim 50 \mathrm{Myr}$ is the timescale for $z$ oscillations in the potential of the Galaxy. This is more than two orders of magnitude lower than the offsets measured for some sGRBs in galaxies with similar stellar mass to that of the Milky Way (see table 2). Therefore, if BNSs are born close enough to the Galactic plane, that their motion is dominated by the Galactic potential, the expected distribution of kicks does not naturally account for large off-sets. To demonstrate the latter point we plot in Fig. 2 the vertical 
offset distribution of BNS systems at the time of merger, as resulting from a Monte Carlo simulation, in which the birth conditions of the binaries are informed by constraints from the Galactic BNS population and the systems' motion in the Galaxy's potential is then calculated with galpy ${ }^{1}$ (Bovy 2015) using the MWPotent ial2 014 Galactic potential (full details of the Monte Carlo calculation are given in appendix A). The results re-affirm the OoM estimate for $z_{\text {typ }}$ above, and the tension with sGRB offsets.

Of course, if BNS systems can be formed far away from the centers of their host galaxies, large off-sets can be naturally explained with no need for invoking kicks. ${ }^{2}$ In such a situation, the offsets of BNS mergers should correlate with the spatial extent of star formation in their host galaxies. This too is demonstrated in the results of our Monte Carlo calculation for the Milky Way presented in Fig. 2. This also naturally leads to a systematic difference between the offsets of BNS mergers in early versus late time galaxies as seen in observations (see Section 3).

\subsection{Offset differences due to differences in propagation times}

Early-type galaxies have very old stellar populations, typically 5-13 Gyr old, compared with the younger stellar populations in late-type galaxies. One might therefore suggest that in early-type galaxies, $\mathrm{Ca}$-rich $\mathrm{SNe}$ or short-GRB progenitors which were kicked early-on would take a longer time to propagate in their host galaxies, and would attain larger offsets. However, as we also discussed above in the context of short-GRBs, if the progenitors were ejected with kicks lower than the galaxy escape velocity they would not escape the galaxy, but rather oscillate in its potential. Since the typical dynamical time-scales in galaxies (of the order of tens of Myrs) are far shorter than the typical ages of the stellar population in early-type galaxies, they would later explode at some random phase during the oscillation. Consequently, their offsets would generally not correlate with the age of the stellar population. In Fig. 3, we compare the offsets following short $(0.1 \mathrm{Gyr})$ and long $(10 \mathrm{Gyr})$ propagation times in a Milky Way potential and applying strong kicks, consistent with those inferred for the general pulsar population (Hobbs et al. 2005) (note that these are much stronger than kicks inferred for the Galactic BNS population, as discussed in Section 4.2). The results demonstrate that the propagation time following strong kicks cannot explain the difference between early- and late-type galaxies.

If kicks were larger than the escape velocity (and not fine-tuned to be very close to the escape velocity) than at the age of early-type galaxies, the progenitors would travel Mpcs before exploding, and not show up at the much smaller offsets observed.

We therefore conclude that age differences considered with some natal kicks are not likely to explain the differences in the offsets in early- versus late-type galaxies. In contrast, these differences are

\footnotetext{
${ }^{1}$ http://github.com/jobovy/galpy

${ }^{2}$ We note, however, that at large offsets, the galactic potentials can become sufficiently weak such that the BNS can travel unimpeded, even with a very small change to its center of mass. The BNS will then cover a distance $\approx t_{\mathrm{mrg}} \Delta v_{\mathrm{cm}}$ before merging (where $t_{\mathrm{mrg}}$ is the delay time between BNS formation and merger). Recently, (Beniamini \& Piran 2019) have shown that the lifetime of the pulsars in Galactic BNS systems are sufficiently short, that the delay times may be directly inferred from the currently observed time until merger in those systems. The result is a rather steep delay time distribution in which at least $40-60$ per cent $(10-20$ per cent $)$ of systems were formed with delays shorter than $1 \mathrm{Gyr}(100 \mathrm{Myr})$. Therefore, even in this case BNS systems may not move very far (compared to their offset at birth) before eventually merging.
}

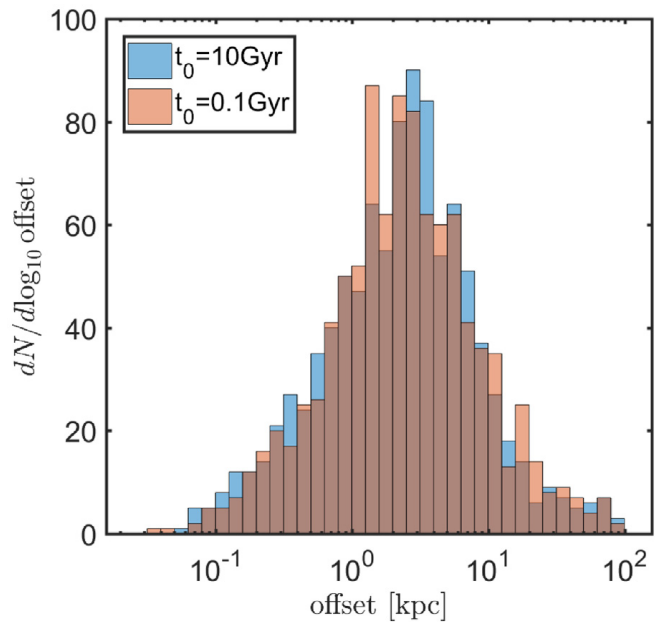

Figure 3. Results of a Monte Carlo simulation calculating the total offsets of systems kicked (assuming the widely used Hobbs velocity-kick distribution; Hobbs et al. 2005) in the Milky Way potential (see Section A for details), for systems which were allowed to propagate for either short, $0.1 \mathrm{Gyr}$, or long, 10 Gyr, time-scales. As can be seen the overall distributions are indistinguishable since the propagation time-scales exceed the dynamical oscillation timescales in the galaxy, and the systems' offset depends on the random phase during their back and forth oscillation in the galaxy.

naturally consistent with the observed differences in stellar mass distributions in early- versus late-type galaxies.

\section{SUMMARY}

Ca-rich SNe and sGRBs are two groups of transients, of which members show an extended spatial distribution in their host galaxies. In particular, a large fraction of these transients are found at large $(>10 \mathrm{kpc})$ from the nuclei of their galaxy hosts, and apparently far from any observed underlying stellar population, which could host their progenitors. These perplexing findings motivated various solutions. These include models in which the progenitors of such transients received large velocity kicks allowing them to migrate to remote positions where they exploded; or that the progenitors originated in globular clusters, the distribution of which extends far into the halo. However, such solutions are difficult to reconcile with other properties of the transients and their suggested progenitors, as we discussed above.

In this study, we analyzed the distributions of the distance offsets of Ca-rich supernovae and sGRBs from their host galaxies. We pointed out that stacked-images and deep-imaging of early- and late-type galaxies show different stellar mass distribution, and that early-type galaxies have very extended stellar mass, with a large fraction, typically the majority of the stellar mass residing in the halo beyond $10 \mathrm{kpc}$. Therefore, in contrast to previous studies, we divided the offset distributions between early- and late-type galaxies. We showed that the offsets in early-type galaxies extend farther than that in late-type galaxies. Moreover, the fractions of transients in the halo $(>10 \mathrm{kpc})$ versus the central parts $(<10 \mathrm{kpc})$ are consistent with the observed distinctly different halo to central parts stellar mass ratios in early-type and late-type galaxies (D'Souza et al. 2014; Huang et al. 2018).

We also studied the expected offset distribution, given the kinematics in the Galactic potential, for progenitors that receive velocity kicks and show that no large kicks are required in order to explain the observed offsets. A related argument, in a slightly different context, 
was made by Mandel (2016), who has shown that no large black hole velocity kicks are needed to explain the observed positions and velocities of the observed black hole low mass X-ray binaries in the Galaxy. The apparent disagreement of our results with previous estimates of large kicks from significantly offset sGRBs (Zevin et al. 2020) could be driven by the host galaxy properties and their evolution. In particular, if the amount of stellar material at larger offsets is underestimated (which as we discuss here, is expected to be the case in early-type galaxies, when considering the half light radii as a proxy for the stellar content), one would infer artificially larger kicks by applying a forward modelling approach.

We conclude that the progenitors of both Ca-rich SNe and sGRBs do not require large velocity kicks, nor do their progenitor need to form in globular clusters. Rather, their offset distributions are generally consistent with the underlying stellar mass distribution of their host galaxies. The appearance of large offsets is then consistent with old stellar progenitors for significant fractions of these transients.

Finally, we note that since stacked/ultra-deep imaging show that early-type galaxies are more extended than late-type galaxies, any study of other transients' offset distribution, such as FRBs should account for the host galaxy-type. In fact, the recent finding of FRBs at large offsets (Ravi et al. 2019), does not require their progenitors to have had natal kicks, and we expect FRBs with large offsets to be mostly identified in early-type galaxies.

\section{ACKNOWLEDGEMENTS}

We would like to thank the referee for thoughtful and constructive comments that improved the manuscript. HBP acknowledges support for this project from the European Union's Horizon 2020 research and innovation program under grant agreement No 865932-ERC$\mathrm{SNeX}$ and the Kingsley distingushed visitor program at Caltech. The research of PB was funded by the Gordon and Betty Moore Foundation through Grant GBMF5076.

\section{DATA AVAILABILITY}

The simulations underlying this article will be shared on reasonable request to the corresponding author.

\section{REFERENCES}

Abbott B. P. et al., 2017, ApJ, 848, L12

Beniamini P., Piran T., 2016, MNRAS, 456, 4089

Beniamini P., Piran T., 2019, MNRAS, 487, 4847

Beniamini P., Hotokezaka K., Piran T., 2016, ApJ, 829, L13

Bloom J. S., Kulkarni S. R., Djorgovski S. G., 2002, AJ, 123, 1111

Bovy J., 2015, ApJS, 216, 29

Burgay M. et al., 2003, Nature, 426, 531

Cameron A. D. et al., 2018, MNRAS, 475, L57

D'Souza R., Kauffman G., Wang J., Vegetti S., 2014, MNRAS, 443, 1433

Da Rocha C., Ziegler B. L., Mendes de Oliveira C., 2008, MNRAS, 388, 1433

Dall'Osso S., Piran T., Shaviv N., 2014, MNRAS, 438, 1005

De K. et al., 2018, ApJ, 866, 72

De K. et al., 2020, ApJ, 905, 58

De K., Fremling U. C., Gal-Yam A., Yaron O., Kasliwal M. M., Kulkarni S. R., 2021, ApJ, 907, L18

Dessart L., Hillier D. J., 2015, MNRAS, 447, 1370

Eichler D., Livio M., Piran T., Schramm D. N., 1989, Nature, 340, 126

Foley R. J., 2015, MNRAS, 452, 2463
Fong W., Berger E., 2013, ApJ, 776, 18

Fong W. et al., 2017, ApJ, 848, L23

Fong W. et al., 2021, ApJ, 906, 127

Gal-Yam A., Maoz D., Guhathakurta P., Filippenko A. V., 2003, AJ, 125, 1087

Gallazzi A., Charlot S., Brinchmann J., White S. D. M., Tremonti C. A., 2005, MNRAS, 362, 41

Gompertz B. P., Levan A. J., Tanvir N. R., 2020, ApJ, 895, 58

Gonzalez A. H., Zaritsky D., Zabludoff A. I., 2007, ApJ, 666, 147

Goodman J., 1986, ApJ, 308, L47

Hobbs G., Lorimer D. R., Lyne A. G., Kramer M., 2005, MNRAS, 360, 974

Huang S. et al., 2018, MNRAS, 480, 521

Jacobson-Galán W. V. et al., 2020, ApJ, 898, 166

Jacobson-Galán W. V. et al., 2021, ApJ, 908, L32

Kasliwal M. M. et al., 2012, ApJ, 755, 161

Kramer M. et al., 2006, Science, 314, 97

Lazarus P. et al., 2016, ApJ, 831, 150

Li H. et al., 2018, MNRAS, 476, 1765

Lunnan R. et al., 2017, ApJ, 836, 60

Lyman J. D., James P. A., Perets H. B., Anderson J. P., Gal-Yam A., Mazzali P., Percival S. M., 2013, MNRAS, 434, 527

Lyman J. D., Levan A. J., Church R. P., Davies M. B., Tanvir N. R., 2014, MNRAS, 444, 2157

Lyman J. D., Levan A. J., James P. A., Angus C. R., Church R. P., Davies M. B., Tanvir N. R., 2016, MNRAS, 458, 1768

Mandel I., 2016, MNRAS, 456, 578

Maoz D., Mannucci F., Nelemans G., 2014, ARA\&A, 52, 107

Martinez J. G. et al., 2017, ApJ, 851, L29

Maund J. R., Smartt S. J., 2005, MNRAS, 360, 288

Metzger B. D., 2012, MNRAS, 419, 827

Milisavljevic D. et al., 2017, ApJ, 846, 50

Nugent A. E. et al., 2020, ApJ, 904, 52

O'Connor B. et al., 2021, MNRAS, 502, 1279

Paterson K. et al., 2020, ApJ, 898, L32

Perets H. B., 2014, preprint (arXiv:1407.2254)

Perets H. B. et al., 2010, Nature, 465, 322

Perets H. B., Gal-yam A., Crockett R. M., Anderson J. P., James P. A., Sullivan M., Neill J. D., Leonard D. C., 2011, ApJ, 728, L36

Perets H. B., Zenati Y., Toonen S., Bobrick A., 2019, preprint (arXiv:1910.0 7532)

Piran T., Shaviv N. J., 2004, preprint (arXiv:Astrophysics e-prints)

Piran T., Shaviv N. J., 2005, Phys. Rev. Lett., 94, 051102

Ravi V. et al., 2019, Nature, 572, 352

Sand D. J. et al., 2011, ApJ, 729, 142

Sanderson R. E. et al., 2018, ApJ, 869, 12

Sell P. H., Maccarone T. J., Kotak R., Knigge C., Sand D. J., 2015, MNRAS, 450,4198

Shaw R. L., 1979, A\&A, 76, 188

Shen K. J., Kasen D., Weinberg N. N., Bildsten L., Scannapieco E., 2010, ApJ, 715, 767

Shen K. J., Quataert E., Pakmor R., 2019, ApJ, 887, 180

Stovall K. et al., 2018, ApJ, 854, L22

Tauris T. M. et al., 2017, ApJ, 846, 170

Toonen S., Perets H. B., Igoshev A. P., Michaely E., Zenati Y., 2018, A\&A, 619, A53

Urquhart J. S., Figura C. C., Moore T. J. T., Hoare M. G., Lumsden S. L., Mottram J. C., Thompson M. A., Oudmaijer R. D., 2014, MNRAS, 437, 1791

Valenti S. et al., 2014, MNRAS, 437, 1519

Vigna-Gómez A. et al., 2018, MNRAS, 481, 4009

Waldman R., Sauer D., Livne E., Perets H., Glasner A., Mazzali P., Truran J. W., Gal-Yam A., 2011, ApJ, 738, 21

Yuan F., Kobayashi C., Schmidt B. P., Podsiadlowski P., Sim S. A., Scalzo R. A., 2013, MNRAS, 432, 1680

Zenati Y., Perets H. B., Toonen S., 2019, MNRAS, 486, 1805

Zevin M., Kelley L. Z., Nugent A., Fong W.-F., Berry C. P. L., Kalogera V., 2020, ApJ, 904, 190 


\section{APPENDIX: MONTE CARLO SIMULATION OF BNS MERGER VERTICAL OFFSETS}

We apply a Monte Carlo calculation to estimate the distribution of vertical offsets of BNS mergers above the Galactic plane (see also Vigna-Gómez et al. 2018 for a related approach). We note that for the purpose of exploring the effects of kicks in a disc galaxy, the vertical offset is more illuminating than the overall, $\sqrt{R^{2}+z^{2}}$, offset. The reason is that smaller kicks are needed for the amplitude of the oscillations in the Galaxy potential to overcome the initial (relatively narrow) vertical extent of the systems, as compared with the radial scale (in other words, the vertical offset is a more sensitive tool for probing the potential effects of kicks). Naturally, for sufficiently large kicks, the vertical and total offset become comparable.

We begin by drawing the birth locations of the binaries. The systems are assumed to either (i) be born in the Galactic plane (this allows to isolate the effect of kicks from the initial height distribution) or (ii) be drawn from a distribution $d \mathrm{P} / d|z| \propto \exp \left(-|z| / h_{z}\right)$ with $h_{z}=0.3 \mathrm{kpc}$ (matching the adopted MWPotential2014 Galactic potential (Bovy 2015)). These different initial height distributions are used in order to probe the role of kicks for determining the final vertical offset. A realistic distribution of initial offsets in the Milky Way, likely lies between those two extremes, given the tendency of massive stars to be born in a relatively thin disc around the Galactic plane (Urquhart et al. 2014). We point out that the fact that massive stars are more vertically concentrated as compared to the rest of the stellar population in spiral galaxies such as the Milky Way, only serves towards increasing the offset differences between spirals and ellipticals. Indeed, this is in support of the basic picture suggested in the paper.

For either initial height assumption, their Galactic radius is drawn from a distribution $d \mathrm{P} / d r \propto \exp \left(-r / h_{r}\right)$ with $h_{r}=2.6 \mathrm{kpc}$ (again matching MWPotential2014). The systems are initially rotating in the Galactic with their tangential velocity set by the MWPotential2014 rotation curve. Next, we randomize the kicks and mass ejection in the superonova leading to the the birth of the second NS in the binary. Following Beniamini \& Piran (2016) we take lognormal distributions with median values of $\Delta M_{0}, v_{\mathrm{k}, 0}$ respectively and with widths $\sigma_{\Delta M} / \Delta M_{0}=\sigma_{v_{\mathrm{k}, 0}} / v_{\mathrm{k}, 0}=0.5$. As per Beniamini \& Piran (2016), 2/3 of the systems surviving the supernova (and merging within less than a Hubble time) have weak collapses and small mass ejection, $\Delta M_{0}=0.05 M_{\odot}, v_{\mathrm{k}, 0}=5 \mathrm{kms}^{-1}$ while the other $1 / 3$ have values more typical of standard core-collapse supernovae, $\Delta M_{0}=$ $1 M_{\odot}, v_{\mathrm{k}, 0}=160 \mathrm{kms}^{-1}$. We also include an additional amount of ejecta, $\Delta M_{v}=0.1 M_{\odot}$ due to neutrino emission (Beniamini, Hotokezaka \& Piran 2016). The direction of the kicks is assumed to be uncorrelated with the orientation of the binary's orbit. For the latter, we also assume the orbit to be circular before the supernova takes place with the initial separation of the systems following a PL distribution $d N / d a \propto a^{-2.2}$ above $a_{\min }=7.5 \times 10^{10} \mathrm{~cm}$. The latter distribution is consistent with the observed Galactic binaries and reproduces their observed delay time distribution between second NS formation, and BNS merger (Beniamini \& Piran 2019). With these ingredients in place, we self-consistently calculate the resulting orbit in each system, the time between its formation and merger and its center of mass velocity (see Beniamini et al. 2016 for more details). We then propagate the motion of the systems in the Galactic potential using galpy (Bovy 2015) until the time of merger. We repeat this process $10^{3}$ times and record the distribution of heights of merging binaries above the Galactic plane. The results are shown in Fig. 2.

For the purpose of exploring the potential impact of the propagation times in a scenario including substantial kicks, we also run a calculation in which the kick magnitude is taken as a Maxwellian with $\sigma=265 \mathrm{kms}^{-1}$ according to the Hobbs et al. (2005) distribution inferred for regular (i.e. not BNS) pulsars. For this calculation, the ejecta mass is taken as $\Delta M_{0}=1 M_{\odot}$ in all cases. The initial separations are chosen to match two merger times: $0.1 \mathrm{Gyr}$ and 10 Gyr. The results of this calculation are presented in presented in Fig. 3. We emphasize that this calculation is merely a means to demonstrate that the propagation time does not significantly affect the offset, even when the kicks are rather large. The parameters adopted for this example are unlikely to be realistic for true BNS systems.

This paper has been typeset from a $\mathrm{T}_{\mathrm{E}} \mathrm{X} / \mathrm{LT} \mathrm{E} \mathrm{X}$ file prepared by the author. 